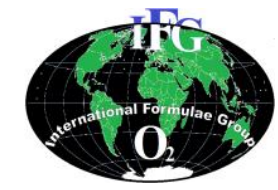

Available online at http://www.ifgdg.org

Int. J. Biol. Chem. Sci. 14(2): 424-439, February 2020

International Journal

of Biological and

Chemical Sciences

ISSN 1997-342X (Online), ISSN 1991-8631 (Print)

Original Paper http://ajol.info/index.php/ijbcs

http://indexmedicus.afro.who.int

\title{
Variabilité des traits fonctionnels des espèces arborescentes dans la reconstitution de la végétation du Parc National d'Azagny (Côte d'Ivoire)
}

\author{
Adjo Estelle Genevieve ADIKO ${ }^{1 *}$, Stéphanie Diane Konan HOUPHLET ${ }^{2}$, \\ Sèdoami Flora DOGBO ${ }^{1,3}$, Bi Tra Aimé VROH ${ }^{1}$, Djaha KOUAME², \\ Zoro Bertin GONE BI ${ }^{1,4}$, Anthelme GNAGBO ${ }^{2}$, Jean-Claude Koffi $\mathrm{BENE}^{2}$ et \\ Constant Yves ADOU YAO ${ }^{1,4}$
}

\footnotetext{
${ }^{1}$ Equipe BioValSE, Laboratoire de botanique, UFR Biosciences, Université Félix Houphouët-Boigny, 22 BP 582 Abidjan 22, Côte d'Ivoire.

${ }^{2}$ Equipe BioValSE, UFR Environnement, Université Jean Lorougnon Guédé, BP 150 Daloa, Côte d'Ivoire.

${ }^{3}$ Laboratoire de Biomathématiques et d'Estimations Forestières (LABEF), Faculté des Sciences Agronomiques- Université d'Abomey-Calavi, 04 BP 1525, Cotonou, Bénin.

${ }^{4}$ Centre Suisse de Recherches Scientifiques en Côte d'Ivoire ; 01 BP 1303 Abidjan 01, Côte d'Tvoire.

*Auteur correspondant ; E-mail: genevieveadiko@gmail.com ; Tel : (+225) 57975434 /(+225) 89190003
}

\section{REMERCIEMENTS}

Nous adressons nos remerciements au programme Sud Expert Plante et Développement Durable (SEP2D) pour avoir accepté de financer ces travaux de recherche.

\section{RESUME}

Dans le Parc National d'Azagny, des plantations de cultures pérennes ont été abandonnées, certaines détruites de même que des habitats successivement en 1986 et 2000. Ainsi donc, l'ensemble de la végétation du parc est constitué de reliques de forêts anciennes, de forêts secondaires et de jachères d'âge différents. La présente étude a évalué les changements des traits fonctionnels des espèces végétales arborescentes dans différentes formations du Parc National d'Azagny. Il s'est agi, spécifiquement de : décrire les traits de vie des espèces arborescentes et comparer la composition fonctionnelle entre les forêts anciennes, les forêts secondaires et les jachères de 19 et 33 ans. Le type foliaire, le mode de dissémination et la tolérance à la lumière ont été renseignés pour les individus arborescents de diamètre $\geq 2,5 \mathrm{~cm}$ recensés dans des parcelles de 0,25 ha. Le spectre fonctionnel des espèces a été déterminé et des tests de Kruskal-Wallis ont permis de comparer la composition fonctionnelle entre ces formations. L'étude des spectres fonctionnels réalisée sur 1972 individus d'arbres, répartis entre 127 espèces et 46 familles a montré une dominance des espèces à feuilles mésophylles et macrophylles, des espèces sciaphiles et de la zoochorie dans chacun des biotopes. Les tests de Kruskal-Wallis ont montré des différences significatives dans la composition fonctionnelle des habitats au niveau des modalités de traits mégaphylles, pionnières, barochores et zoochores. Ces travaux révèlent un retour des processus écologiques initiaux, donc une bonne reconstitution des milieux perturbés, qui sera facilitée par un renforcement des mesures de protection.

(C) 2020 International Formulae Group. All rights reserved.

Mots clés: Utilisation des terres, succession secondaire, diversité fonctionnelle, Parc National d'Azagny, Côte d'Ivoire. 


\title{
Variability of functional traits of tree species in the recovery of vegetation in Azagny National Park (Côte d'Ivoire)
}

\begin{abstract}
In Azagny National Park, plantations of perennial crops have been abandoned, some destroyed as well as habitats successively in 1986 and 2000. Thus, all the vegetation of the park consists of relics of ancient forests, secondary forests and fallows of different ages. This study evaluated changes in the functional traits of tree species in different formations of Azagny National Park. Specifically, it was to describe the life traits of tree species and to compare the functional composition between old-growth forests, secondary forests and fallows aged 19-33. Leaf type, diaspores dispersion and light tolerance were reported for tree individuals with $\mathrm{dhp} \geq 2.5 \mathrm{~cm}$ in 0.25 ha plots. The functional spectra of the species were determined and Kruskal-Wallis tests were used to compare the functional composition between these different formations. The study of functional spectra carried out on 1972 individuals of trees, distributed among 127 species and 46 families, showed a dominance of mesophyllous and macrophyllous leaf species, shade-tolerant species and zoochory in each of the biotopes. The Kruskal-Wallis tests showed significant differences in the functional composition of habitats at the level of megaphyllous, pioneer, barochory and zoochory traits. This work reveals a return of the initial ecological processes, thus a good reconstitution of the disturbed environments, which will be facilitated by a reinforcement of the protective measures.
\end{abstract}

(C) 2020 International Formulae Group. All rights reserved.

Keywords: Land use, secondary succession, functional diversity, Azagny National Park, Côte d'Ivoire.

\section{INTRODUCTION}

Les forêts tropicales humides renferment plus de la moitié des espèces vivantes (Puig, 2001). Aussi, ces forêts fournissent de nombreux services à l'homme (la régulation du climat, de l'eau, de l'air, de l'érosion, l'approvisionnement en nourriture, en eau, en plantes médicinales, lieu de récréation, de tourisme). Cependant, face à l'accroissement démographique, l'exploitation des ressources, la quête permanente de terres cultivables, on assiste à une destruction voire une disparition de ces forêts au fil des temps (Mirabel, 2018).

En vue de remédier à ce phénomène, au Sommet de la Terre à Rio en 1992, l'accent a été mis sur le renforcement de la protection, de la gestion durable et de la conservation de ces forêts qui rendent de nombreux services écosystémiques (Flores, 2005).

La Côte d'Ivoire n'est pas en reste de cette prise de conscience de la nécessité de gestion des ressources naturelles. En effet, un réseau de six réserves naturelles et de huit parcs nationaux est reparti sur l'ensemble de son territoire (UICN/BRAO, 2008).
Malgré cette volonté de conservation, force est de constater l'existence de pratiques anthropiques (prélèvement de produits forestiers non ligneux et ligneux, activités agricoles) dans les aires protégées ivoiriennes. Ce constat a été fait dans le Parc National d'Azagny situé au sud de la Côte d'Ivoire à l'intérieur duquel, les formations secondaires (forêts secondaires et jachères) et les forêts anciennes occupent actuellement, respectivement $24,41 \%$ et $19,79 \%$ de la superficie totale (Konan, 2017). Ces formations secondaires résultent de l'abandon en 1986 et en 2000 lors d'opérations de déguerpissement menées par l'OIPR des plantations de cultures pérennes telles que les cultures de cacaoyers (Theobroma cacao L.), de caféiers (Coffea canephora A. Froehner), de palmiers à huile (Elaeis guinnensis Jacq), d'hévéas (Hevea brasiliensis (Kunth) Müll. Arg) et de cocotiers (Cocos nucifera L.), périodes pendant lesquelles plusieurs plantations ont été complètement détruites de même que des habitats humanisés. L'étude réalisée par Koffi et al. (2015) a montré que l'installation de ces cultures a eu un impact sur la diversité 
floristique en fonction du précédent cultural et de l'âge d'abandon. Au-delà de la diversité floristique, ce genre de perturbations liées aux activités agricoles, agissent sur le fonctionnement des écosystèmes qui inclus en réalité les services écosystémiques dont dépend l'homme pour sa survie (Giller et al., 2004). Le fonctionnement des écosystèmes est l'ensemble des propriétés et/ou processus biotiques et abiotiques au sein des écosystèmes, comme par exemple le recyclage ou la production de biomasse, est déterminé à travers la diversité fonctionnelle qui représente la gamme et la distribution des valeurs de traits fonctionnels dans la communauté (Diaz et Cabido, 2001). Les traits fonctionnels qui peuvent être catégoriels (par exemple le type de reproduction) ou continus (par exemple la taille), sont des caractéristiques morphologiques,

biochimiques, physiologiques, structurelles, phénologiques ou comportementales exprimées en phénotypes mesurables au niveau de l'individu d'une espèce (Violle et al., 2007). L'étude des traits biologiques est une approche complémentaire à la classification taxonomique permettant d'interpréter les effets des perturbations anthropiques (Payeur, 2010). Elle permet de comprendre le fonctionnement des écosystèmes de même que les stratégies adaptatives et la réponse des espèces face à ces pressions. En effet, au cours de la succession secondaire, s'effectue un remplacement des espèces (Kassi et al., 2011) qui est souvent expliqué en termes d'adaptation des espèces à des environnements lumineux changeants (Lohbeck, 2014). Or ce sont ces stratégies adaptatives qui assurent le maintien de l'espèce en plus des actions de conservation. Cependant, les études dans ce domaine demeurent encore insuffisantes surtout dans les forêts ivoiriennes comme celle du parc national d'Azagny qui a subi de fortes pressions. D'où cette étude, qui a pour objectif d'évaluer les changements des traits fonctionnels des espèces végétales entre reliques de forêts anciennes, forêts secondaires et jachères d'âges différents du
Parc National d'Azagny. Spécifiquement, il s'est agi de: (1) déterminer la richesse floristique arborescente des forêts anciennes, des formations secondaires issues de coupe et les jachères de cultures pérennes; (2) déterminer les traits biologiques des espèces constituant le cortège floristique arborescent des forêts anciennes, des formations secondaires issues de coupe et les jachères de cultures pérennes; (3) comparer la diversité fonctionnelle entre les forêts anciennes, les forêts secondaires et les espaces anciennement cultivés.

\section{MATERIEL ET METHODES Site d'étude}

L'étude s'est déroulée dans le Parc National d'Azagny (PNA). Ce parc, au Sud de la Côte d'Ivoire, (Figure 1), s'étend sur une superficie de 21.850 ha. C'est une presqu'île délimitée par le fleuve Bandama à l'Ouest, le canal d'Azagny au Sud, la lagune Ébrié à l'Est et au Nord, par les plantations industrielles et villageoises.

Une mosaïque de formations végétales y est rencontrée (Gnagbo et al., 2016) :

- des forêts anciennes sur terre ferme dont la strate émergente renferme des espèces ligneuses telles que: Strombosia pustulata Oliv., Diospyros sanza-minika A.Chev, Piptadeniastrum africanum (Hook.f.) Brenan, Lophira alata Banks ex Gaertn.f.

- des formations secondaires (forêts secondaires et plantations de cultures pérennes abandonnées ou jachères) qui sont en état de reconstitution. Les forêts secondaires ont une structure intermédiaire entre celle des forêts anciennes et des jachères. Elles sont issues de la destruction complète en 1986 de certaines plantations introduites clandestinement, des habitats ou des activités de prélèvement de produits forestiers ligneux. Les jachères sont constituées de cacaoyères et/ou caféières, d'hévéaculture, des cultures de palmier à huile et de cocoteraies abandonnées en 1986 et en 2000. À la suite de cet abandon des activités agricoles, ces espaces se sont transformés aujourd'hui en des jachères de 19 et 33 ans.

- des formations marécageuses, rencontrées dans les dépressions. 
- des savanes littorales ou côtières sur terre ferme ou sur un sol inondé, dominées par Borassus aethiopum Mart., Phoenix reclinata Jacq. pour les savanes sur terre ferme et par Echinochloa pyramidalis (Lam.) Hitchc. \& Chase et Mariscus flabelliformis Kunth pour les savanes sur sol inondé.

- des mangroves présentent sur les rives de la lagune Ebrié et du canal d'Azagny, dominées par les palétuviers Rhizophora racemosa G.F.W. et Avicennia germinans (L.) L.

\section{Méthodes de collecte des données Inventaire floristique}

Dans le Parc National d'Azagny, des relevés de surface de 0,25 ha $(50 \mathrm{~m}$ x $50 \mathrm{~m})$ ont été réalisés de mai 2018 à mars 2019 dans les jachères de 19 et 33 ans issues des cultures de cacaoyers, de palmiers à huile, de caféiers et d'hévéa, dans les formations secondaires reconstituées depuis 1986 à la suite de la destruction de certaines cultures pérennes, des habitats ou des activités de prélèvement de produits forestiers ligneux et enfin, dans les forêts anciennes sur terre ferme. La présente étude a porté uniquement sur les forêts anciennes sur terre ferme, les forêts secondaires et les jachères du PNA. Les forêts secondaires et les espaces anciennement cultivés ont été choisis sur la base du niveau d'anthropisation qui était très élevé dans ces zones. Quant aux forêts anciennes, elles ont servi de milieu témoin.

À l'intérieur de chaque parcelle, tous les individus arborescents ayant un diamètre à hauteur de poitrine (dbh) pris à $1,30 \mathrm{~m} \mathrm{du}$ sol supérieur ou égal à $2,5 \mathrm{~cm}$ ont été recensés. Ces espèces ont été identifiées sur la base de consultation d'ouvrage notamment le guide de terrain des ligneux des forêts denses humides de l'Afrique de l'Ouest de Hawthorne et Jongkind (2006) et par comparaison aux échantillons de l'Herbier du Centre National de Floristique de Côte d'Ivoire. Au total, 21 parcelles ont été installées dans ces formations réparties comme suit : cinq (05) en forêts anciennes sur terre ferme, neuf (09) en forêts secondaires et sept (07) en jachères.

\section{des espèces \\ Identification des traits fonctionnels}

Pour caractériser les espèces après une perturbation, les traits morphologiques, les traits d'histoire de vie et les traits de régénération sont couramment utilisés (Brakchi-Ouakour et al., 2015 ; Mekideche et al., 2018). Dans cette étude, nous avons choisi trois (03) types de trait. Les traits foliaires ont été choisis dans le cadre des traits morphologiques, le mode de dissémination comme trait de régénération. Comme traits d'histoire de vie, nous avons choisi les guilds des espèces qui rassemblent un ensemble de traits biologiques relatifs à la biologie de la reproduction, à l'installation, à la croissance et à la physiologie des espèces (Tableau 1). Les guilds traduisent le processus de recrutement des espèces selon leur tolérance à la lumière.

Dans le processus de succession, Bangirinama et al. (2010) ont montré que les traits foliaires et le mode de dissémination sont des indicateurs de la dynamique postculturale.

Aussi, ce sont des traits faciles à mesurer car elles étaient accessibles dans la littérature pour la plupart des espèces recensées. Pour certaines espèces, dont les données sur les types foliaires n'étaient pas disponibles dans la littérature, la détermination de ceux-ci s'est faite sur le terrain par la mesure de la surface limbaire de la feuille adulte selon le protocole de Raunkiaer (1934) revue par Mouton (1966).

\section{Méthodes d'analyse des données}

L'analyse des données a concerné pour chaque type d'habitat, la richesse floristique, les proportions des modalités de traits en fonction des espèces recensées et enfin la diversité fonctionnelle des biotopes.

La richesse floristique a consisté à déterminer la richesse spécifique et la répartition des espèces en genres et familles botaniques.

Les proportions des modalités de traits (Tableau 1) en fonction des espèces recensées 
ont été estimées pour chaque biotope. Cette estimation a permis de faire ressortir les modalités de traits exprimés par les espèces.

La diversité fonctionnelle a été évaluée au travers de la composition fonctionnelle. Cette composition fonctionnelle représente l'abondance relative des traits fonctionnels des espèces présentes dans un écosystème donné (Diaz et Cabido, 2001). Elle permet de décrire la variation spatio-temporelle des traits (Klais, 2016). Celle-ci est, le plus souvent, estimée par un indice notamment CWM (Community-Weighted Means) qui représente la moyenne de la valeur de trait pondéré par la communauté. L'expression mathématique de cet indice est :

\section{$\mathrm{CWM}=\sum_{1}^{\mathrm{n}}$ pi x trait i}

Dans cette formule, pi représente l'abondance relative de l'espèce $\mathrm{i}$ et trait i la valeur du trait pour l'espèce i. L'abondance relative d'un trait dans un biotope est le rapport du nombre d'individu des espèces ayant un trait donné sur le nombre total des individus présents dans ce biotope, le tout multiplié par 100. C'est un indice utilisé pour évaluer la dynamique des communautés et déterminer l'influence des activités anthropiques passées (Garnier et al., 2007; Brice, 2015). En effet, les pressions anthropiques agissent comme des filtres environnementaux. Les traits les plus abondants, sont ceux qui sont présents chez les espèces qui se sont les plus adaptés. Vu que les valeurs de trait sont catégorielles ou qualitatives et non continues, cette indice (CWM) représente l'abondance relative des espèces ayant un trait donné dans chacun des habitats, la valeur du trait étant considérée comme égal à 1 (Garnier et al., 2007 ; Brice, 2015 ; Klais, 2016).

Analyses statistiques: Des comparaisons d'abondance moyenne des modalités de traits (Tableau 1) entre chaque habitat, ont été effectuées à l'aide du test de Kruskal-Wallis car les conditions de réalisation d'un test paramétrique n'étaient pas respectées. Aussi, un test de comparaison multiple de Dunn a été appliqué en vue de comparer les moyennes deux à deux lorsque les échantillons présentaient une différence de moyenne à un seuil de significativité de $5 \%$. Les logiciels utilisés pour réaliser ces analyses étaient excel version 2013 et xlstat version 2016.

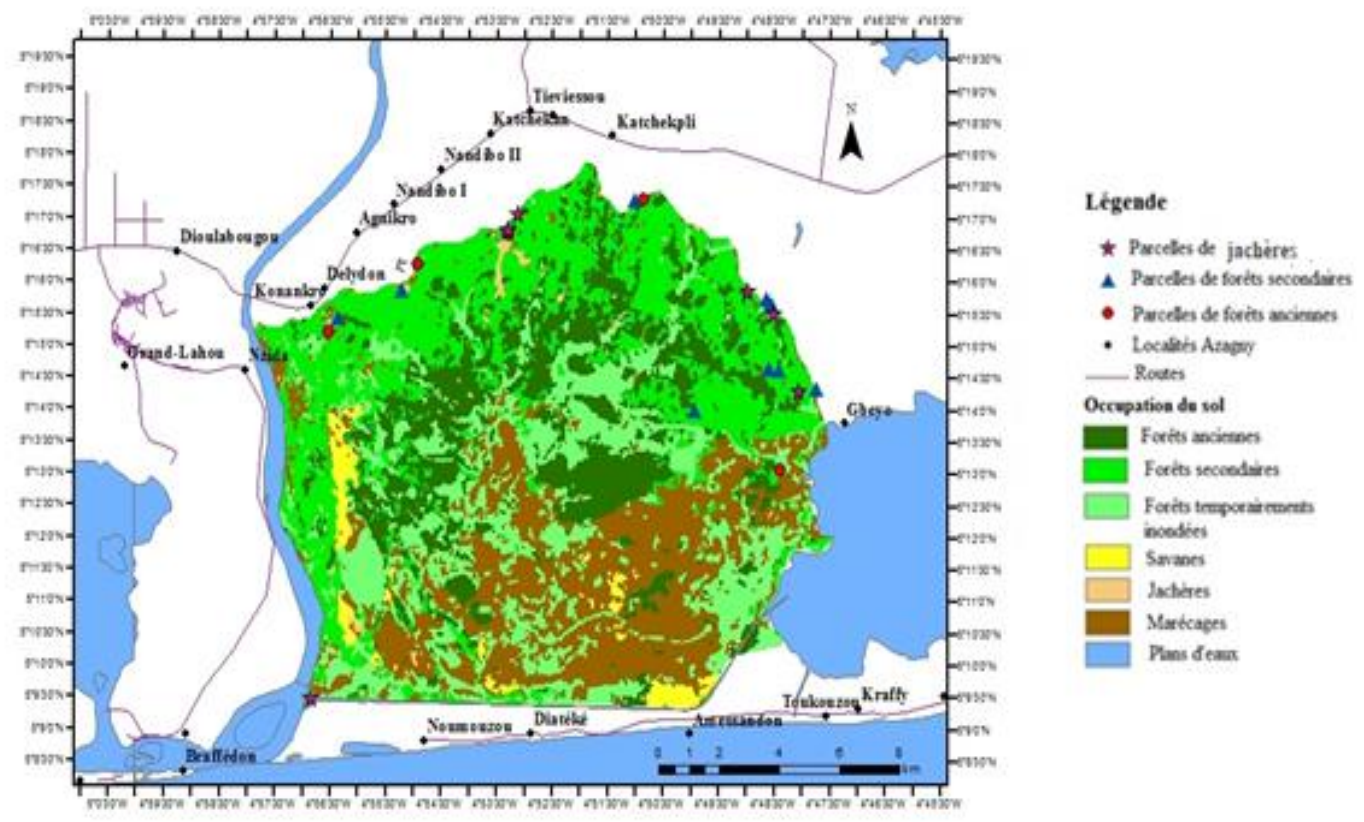

Figure 1: Répartition des parcelles d'inventaires dans les différents biotopes. 
Tableau 1: Traits biologiques et modalités retenus.

\begin{tabular}{|c|c|c|c|}
\hline Catégories de traits & Types de traits & Modalités de trait & Ouvrages consultés \\
\hline Traits morphologiques & Types foliaires & $\begin{array}{l}\text { - Aphylle } \\
\text { - Leptophylle }\left(25 \mathrm{~mm}^{2}\right) \\
\text { - Nanophylle }\left(0,25-2,25 \mathrm{~cm}^{2}\right) \\
\text {-Microphylle }\left(2,25-20,25 \mathrm{~cm}^{2}\right) \\
\text { - Mésophylle }\left(20,25-182,25 \mathrm{~cm}^{2}\right) \\
\text { - Macrophylle }\left(182,25-1640,25 \mathrm{~cm}^{2}\right) \\
\text {-Mégaphylle }\left(0,16 \mathrm{~m}^{2}\right)\end{array}$ & $\begin{array}{l}\text {-Raunkiaer (1934) } \\
\text { - Mouton (1966) }\end{array}$ \\
\hline Trait d'histoire de vie & Ecologie & $\begin{array}{l}\text { - Pionnière } \\
\text { - Héliophile non pionnière } \\
\text { - Espèce d'ombre }\end{array}$ & $\begin{array}{l}\text { - Hall et Swaine } \\
\text { (1981). } \\
\text { - Poorter et al. (2003) }\end{array}$ \\
\hline Traits de régénération & $\begin{array}{l}\text { Mode de } \\
\text { dissémination }\end{array}$ & $\begin{array}{l}\text { - Zoochorie } \\
\text { - Anémochorie } \\
\text { - Barochorie } \\
\text { - Autochorie }\end{array}$ & $\begin{array}{l}\text { - Dansereau et Lem } \\
\text { (1957) } \\
\text { - Sonke (1998) }\end{array}$ \\
\hline
\end{tabular}

\section{RESULTATS}

\section{Richesse floristique des espèces} arborescentes recensées dans les habitats

Les inventaires floristiques ont permis de recenser 1972 individus repartis en 127 espèces. Ces espèces se regroupent en 100 genres au sein de 46 familles dans l'ensemble des habitats.

Les familles les plus dominantes en termes d'espèces sont les Malvaceae (13 espèces, soit 10,24\%), les Fabaceae et les Rubiaceae avec 10 espèces, soit 7,87\% chacune (Figure 2). Les genres les plus représentés sont Cola (06 espèces, soit 4,72\%), Albizia, Celtis, Entandrophragma avec 03 espèces chacune soit 2,36\% (Figure 4). En tenant compte du type d'habitats, la richesse moyenne (par 0,25 ha) la plus élevée est observée au niveau des forêts anciennes $(18,2 \pm 7,918)$ par rapport aux forêts secondaires $(17,44 \pm 10,956)$ et aux jachères $(17 \pm 10,214)$. Le test de Kruskal-Wallis a montré que les différences de moyenne de richesse spécifique ne sont pas significatives $\left(p=0,723 ; X^{2}=0,649\right)$ (Tableau 2).

Les familles les plus importantes dans les forêts anciennes sont les Annonaceae, les Euphorbiaceae et les Malvaceae avec 06 espèces chacune soit 9,84\% (Figure 3). Quant aux genres, les plus importants sont Cola, Xylopia avec 03 espèces chacun soit $4,92 \%$ et Antiaris, Antandrophragma avec 02 espèces chacun soit 3,28\% (Figure 5).

En ce qui concerne les forêts secondaires, les familles les plus importantes sont respectivement les Fabaceae (09 espèces, soit $10,84 \%$ ), les Moraceae (08 espèces, soit $9,64 \%$ ) et les Annonaceae (07 espèces, soit $8,43 \%$ ) (Figure 3). Quant aux genres, il s'agit de Xylopia (03 espèces, soit 3,61\%) et Albizia, Antiaris, Celtis avec 02 espèces chacun soit 2,41\% (Figure 5).

Dans les jachères, les familles les plus importantes sont les Malvaceae (09 espèces, soit 13,43\%), les Annonaceae et les Apocynaceae avec 05 espèces chacune soit $7,46 \%$ (Figure 3). Les genres les plus importants sont Cola (05 espèces, soit 7,46\%), Albizia, Bombax, Celtis avec 02 espèces chacun soit 2,98\% (Figure 5).

\section{Spectre fonctionnel des différents habitats}

Les traits fonctionnels ont été renseignés sur les différentes espèces arborescentes recensées respectivement dans 
les forêts anciennes, les forêts secondaires et dans les jachères.

Trois modes de dissémination ont été identifiés chez les espèces inventoriées en vue d'assurer leur persistance quel que soit l'habitat considéré. Il s'agit de la zoochorie, de l'anémochorie et de la barochoorie. La zoochorie est le mode de dissémination le plus répandu (plus de $60 \%$ des espèces) tandis que la Barochoorie est le moins répandu comme le montre la Figure 6.

Dans l'ensemble des habitats, on rencontre des espèces d'ombre ou sciaphiles, des espèces pionnières et des espèces héliophiles non pionnières. Les espèces d'ombre ou sciaphiles sont les plus importantes (plus de $45 \%$ ) et les espèces nonpionnières tolérantes à l'absence de lumière les moins importantes (plus de 16\%) comme le montre la Figure 7.

Dans l'ensemble des milieux, des espèces à feuilles microphylles, des espèces à feuilles mésophylles, des espèces à feuilles macrophylles et des espèces à feuilles mégaphylles sont rencontrées. Toutefois, les espèces à feuilles mésophylles sont les plus importantes (plus de 65\%) tandis que les espèces mégaphylles et microphylles enregistrent les plus faibles valeurs (3\% chacun) (Figure 8).

\section{Composition fonctionnelle et distribution des traits dans les différents milieux Trait d'écologie des espèces}

Pour ce qui est des traits écologiques des espèces, les forêts anciennes possèdent les plus grandes valeurs moyennes d'abondance relative d'espèces héliophiles non pionnières $(3 \pm 2 \%)$ par rapport aux autres habitats (Figure 9). Le test de Kruskaswallis montre que les différences de moyenne d'abondance relative respective ne sont pas significatives $\left(p=0,248 ; X^{2}=2,787\right)$.

Aussi, l'abondance relative moyenne des espèces sciaphiles dans les forêts anciennes sont plus élevées $(15 \pm 9 \%)$ par rapport aux deux autres habitats (Figure 9). Le test de Kruskas-wallis montre que les différences de moyenne d'abondance relative ne sont pas significatives $\left(p=0,14 ; X^{2}\right.$ $=3,931$ ).

En considérant les espèces pionnières, les jachères ont la plus grande valeur d'abondance relative moyenne $(3,4 \pm 2,4 \%)$ (Figure 9) et le test de Kruskas-wallis montre qu'il existe une différence significative de moyenne d'abondance relative de ce trait entre les forêts secondaires et les jachères $(p$ $=0,047 ; X^{2}=6,130$ ).

\section{Mode de dissémination}

Les forêts anciennes (Figure 10) ont une valeur abondances relative moyennes d'espèces zoochores $(14,9 \pm 9,1 \%)$ la plus élevée, suivies des jachères $(9,3 \pm 4,9 \%)$ et enfin des forêts secondaires $(2,7 \pm 2,1 \%)$. Le test de Kruskal-Wallis révèle une différence significative des abondances relatives moyennes de ce trait entre les différents habitats $\left(p=0002 ; X^{2}=12,944\right)$.

Les espèces anémochores ont l'abondance relative moyenne la plus élevée dans les forêts anciennes $(4,5 \pm 2,9 \%)$ puis dans les jachères $(4,2 \pm 5,5 \%)$ et enfin dans les forêts secondaires $(3,4 \pm 3,4 \%)$ bien que la différence ne soit pas significative ( $\mathrm{p}=0,658$; $\mathrm{X}^{2}=0,836$ ) (Figure 10).

Alors que les espèces barochores ont une abondance relative moyenne significativement plus élevée dans les forêts secondaires $(5 \pm 5,4 \%)$ par rapport aux autres milieux ( $\left.p=0,001 ; X^{2}=14,843\right)$ (Figure 10).

\section{Types foliaires}

Les espèces mésophylles ont une abondance relative moyenne plus élévée dans les forêts anciennes $(16,4 \pm 9,5 \%)$ comparativement aux autres habitats (Figure 11). Cependant le test de Kruskal-Wallis montre que les différences de moyenne d'abondance relative respective entre les types foliaires de ces habitats ne sont pas significatives $\left(P=0,192 ; X^{2}=3,303\right)$.

Les espèces microphylles possèdent les valeurs moyennes d'abondance relative plus élevées dans les forêts anciennes $(0,5 \pm$ $0,7 \%$ ) (Figure 11). Cependant le test de Kruskal-Wallis montre que les différences de moyenne d'abondance relative respective des espèces microphylles entre ces habitats ne 
sont pas significatives $\left(P=0,704 ; X^{2}=\right.$ 0,701).

Les espèces macrophylles possèdent les valeurs moyennes d'abondance relative plus élevées dans les forêts anciennes $(2,8 \pm$ $3,3 \%$ ) (Figure 11). Cependant le test de Kruskal-Wallis montre que les différences de moyenne d'abondance relative respective des espèces macrophylles de ces habitats ne sont pas significatives ( $\left.p=0,328 ; X^{2}=2,227\right)$.
Concernant les espèces mégaphylles, la plus forte valeur d'abondance relative est observée dans les jachères $(0,005 \pm 0,006 \%)$. Le test de Kruskal-Wallis a montré une différence significative des abondances relatives moyennes des espèces mégaphylles entre les jachères par rapport aux forêts secondaires ( $p=0,043 ; X^{2}=6,307$ ) (Figure 11).

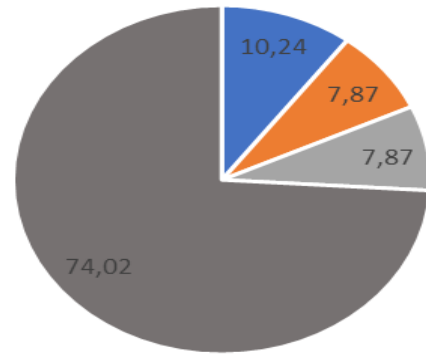

- Malvaceae = Fabaceae $=$ Rubiaceae - Autres familles

Figure 2: Spectre des familles les plus représentatives de l'ensemble des biotopes.
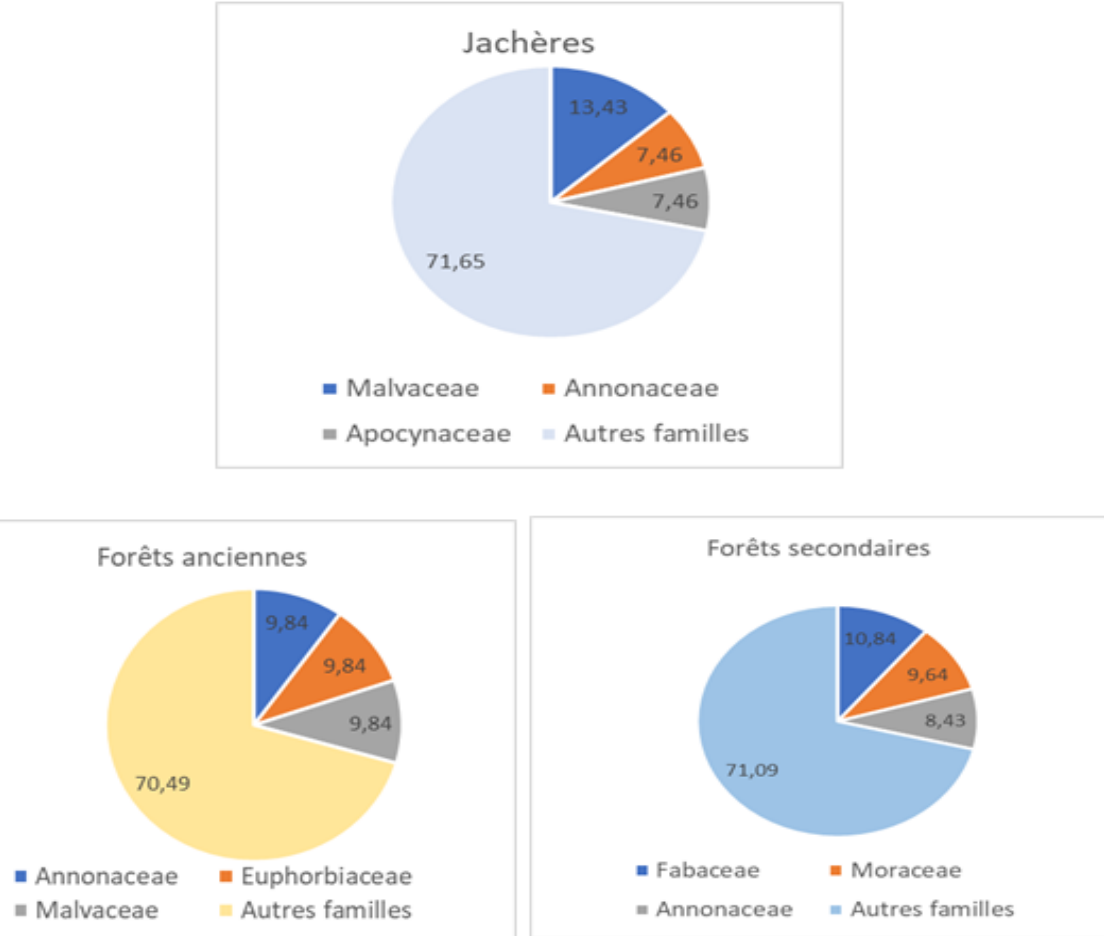

Figure 3: Spectre des familles les plus représentées dans les différents biotopes. 
Tableau 2: Valeurs de la richesse spécifique moyenne des différents habitats.

\begin{tabular}{lllll}
\hline Paramètres floristiques & $\begin{array}{l}\text { Forêts } \\
\text { anciennes }\end{array}$ & $\begin{array}{l}\text { Forêts } \\
\text { secondaires }\end{array}$ & Jachères & Test statistique \\
\hline Richesse spécifique moyenne & $18,2 \pm 7,918$ & $17,44 \pm 10,956$ & $(17 \pm 10,214)$ & $\begin{array}{l}p=0,723 \\
X^{2}=0,649\end{array}$ \\
\hline
\end{tabular}

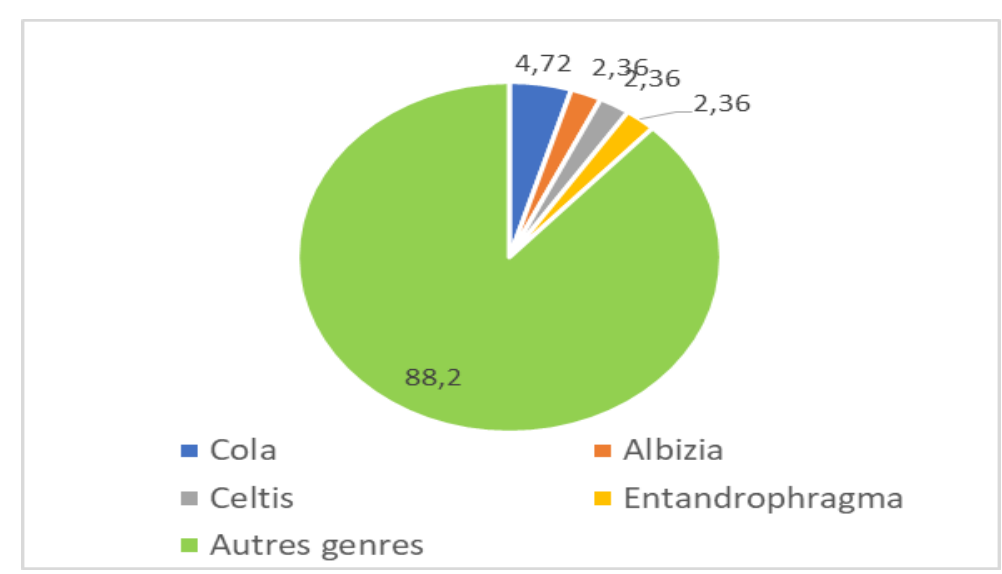

Figure 4: Spectre des genres les plus représentatives de l'ensemble des biotopes.
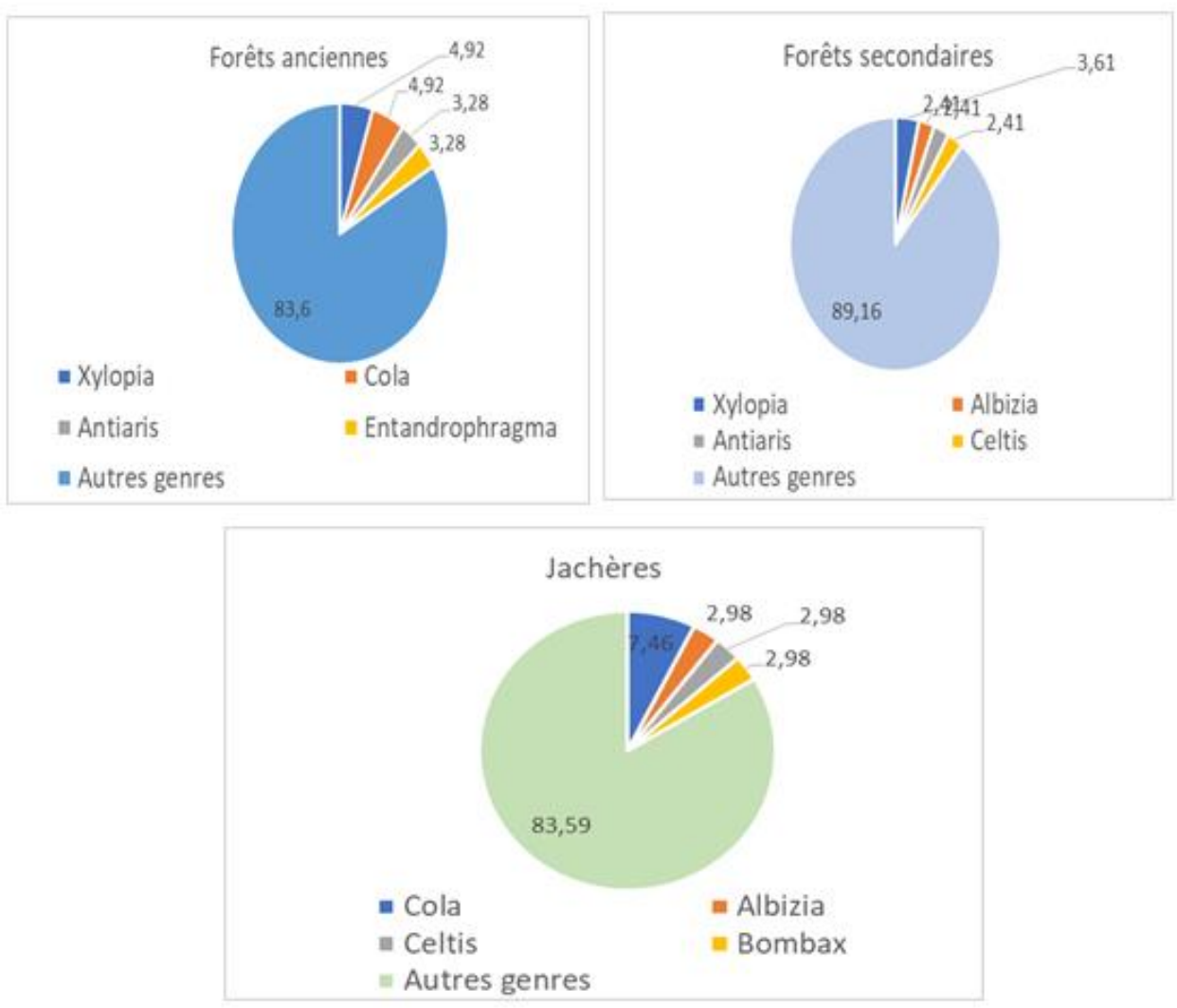

Figure 5: Spectre des genres les plus représentatives dans les différents biotopes. 


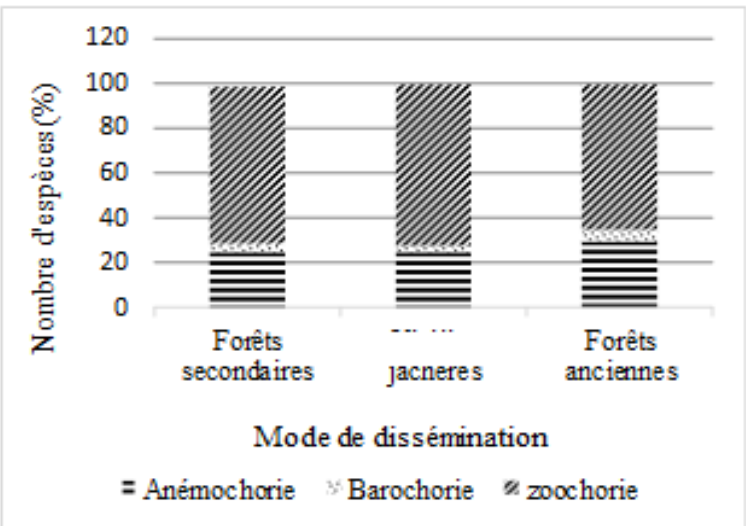

Figure 6: Proportion des modes de dissémination des espèces par biotopes.

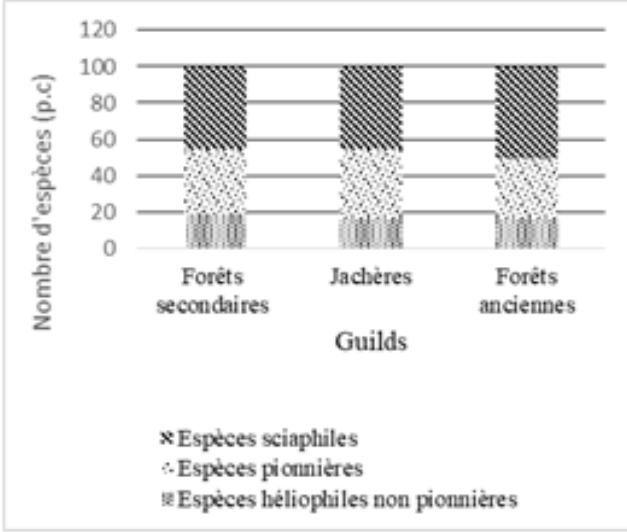

Figure 7: Proportion des Guilds des espèces par biotopes.

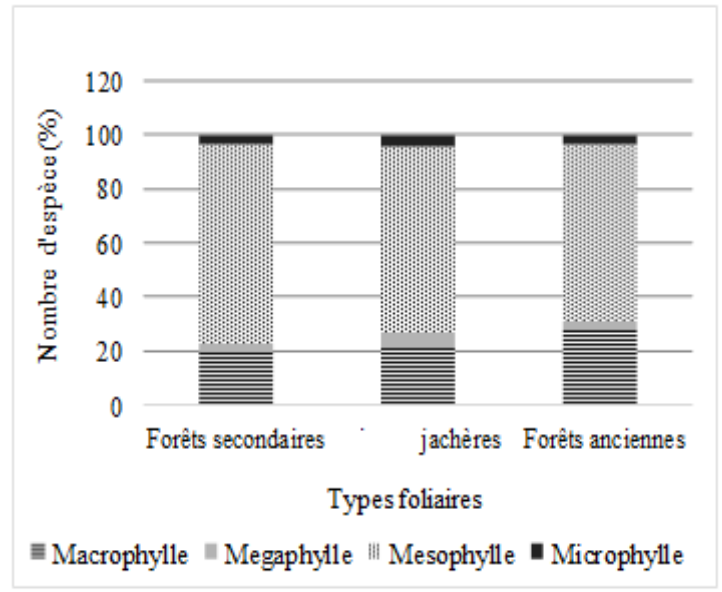

Figure 8 : Proportion des types foliaires des espèces par biotopes.
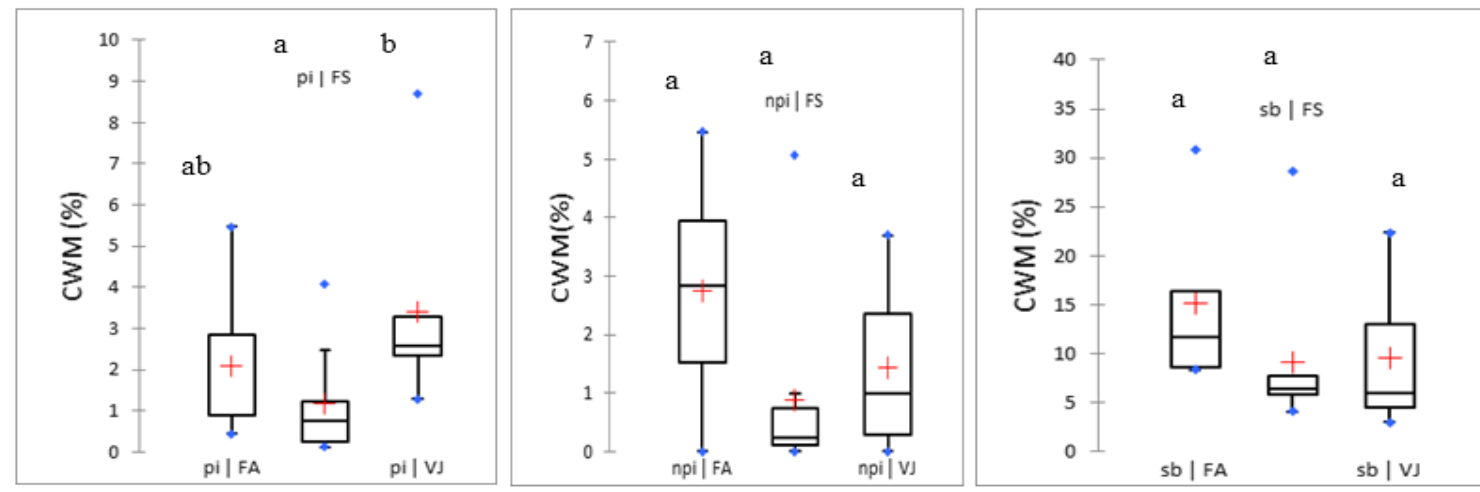

Figure 9: Distribution d'abondance relative des traits d'écologie entre les différents milieux. Légende : Test de Kruskal-Wallis à un seuil de significativité de 5\% : les moyennes affectées de la même lettre (a) ne sont pas significativement différentes. FA : Forêts anciennes, FS : forêts secondaires, JA : Jachères ; Pi : pionnière, npi : nonpionnière, sb : ombre ; CWM : abondance relative des traits, + : Symbole indiquant la valeur moyenne d'abondance relative lue sur l'axe des ordonnées. 

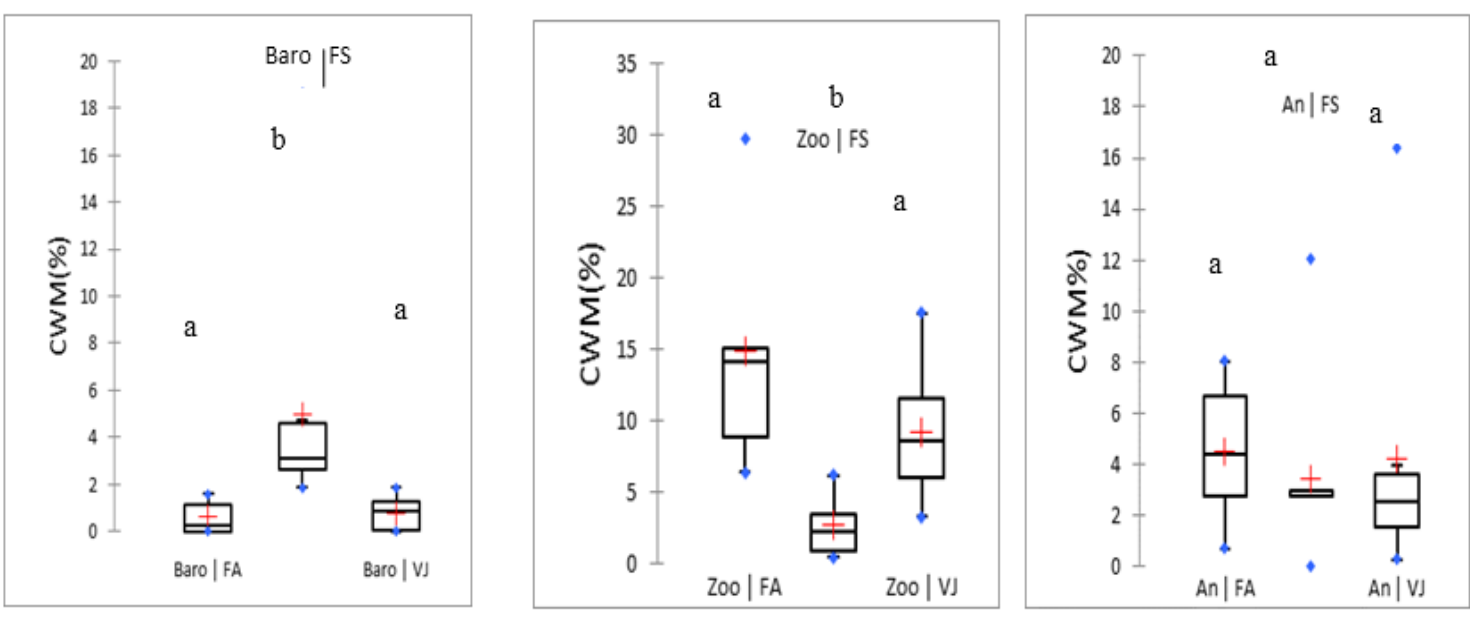

Figure 10: Distribution d'abondance relative des traits de mode de dissémination entre les différents milieux.

Légende: Test de Kruskal-Wallis à un seuil de significativité de 5\%: les moyennes affectées de la même lettre (a) ne sont pas significativement différentes. FA : Forêts anciennes, FS : Forêts secondaires, VJ : Jachères ; Zoo : zoochorie, Baro : barochoorie, An : anémochorie ; CWM : abondance relative des traits, + : Symbole indiquant la valeur moyenne d'abondance relative lue sur l'axe des ordonnées.
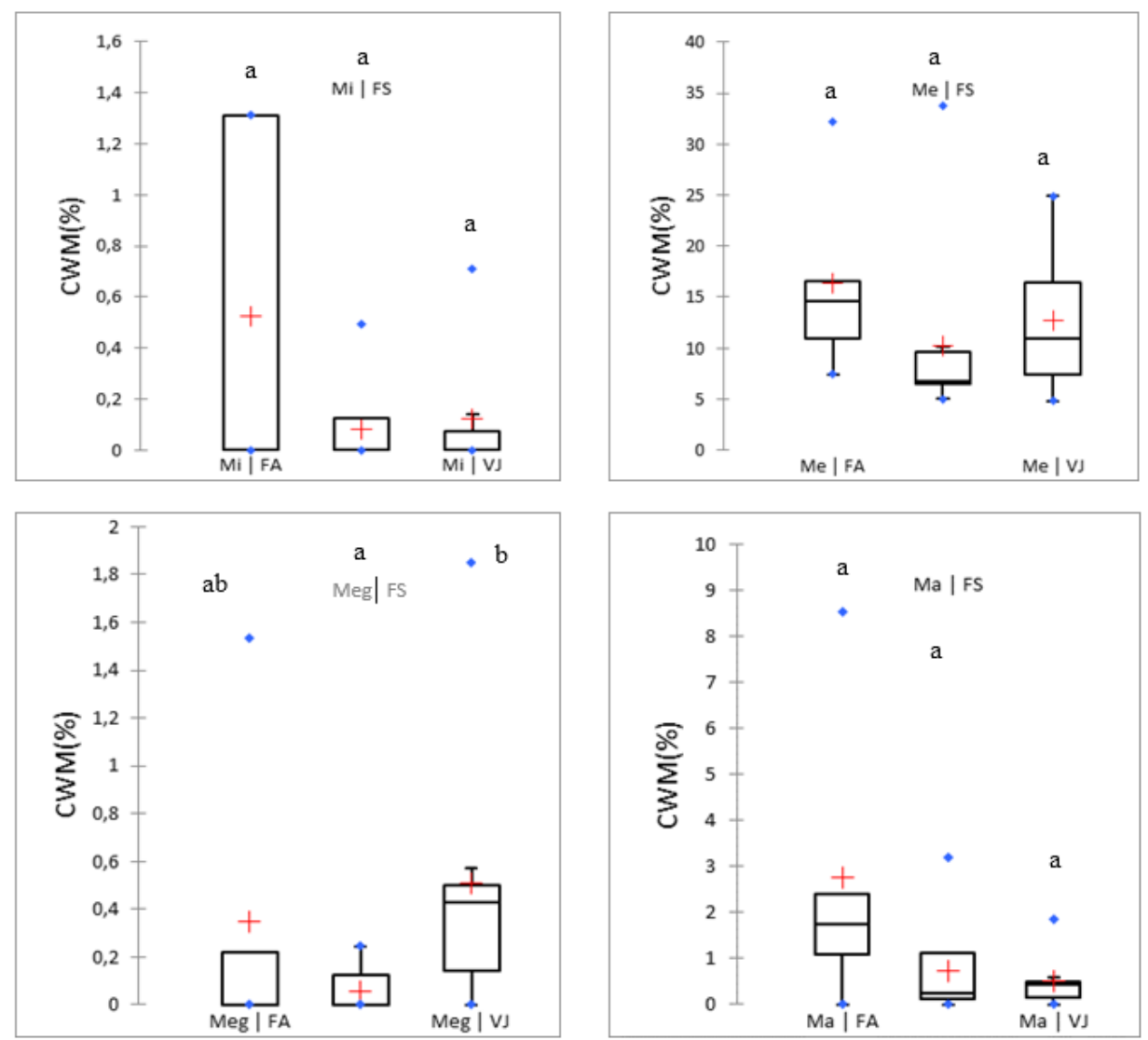

Figure 11: Distribution d'abondance relative des traits foliaires entre les différents milieux.

Légende: Test de Kruskal-Wallis à un seuil de significativité de 5\%: les moyennes affectées de la même lettre (a) ne sont pas significativement différentes; FA : Forêts anciennes, FS : Forêts secondaires, VJ : Jachères, Ma : macrophylle, Meg : mégaphylles, Me : mésophylle, Mi : microphylle; CWM : abondance relative des traits, + : Symbole indiquant la valeur moyenne d'abondance relative lue sur l'axe des ordonnées. 


\section{DISCUSSION}

Cette étude avait pour objectifs de déterminer la richesse floristique, les traits biologiques des espèces arborescentes des forêts anciennes, des formations secondaires issues de coupe et les jachères de cultures pérennes et enfin de comparer la composition fonctionnelle entre les forêts anciennes, les forêts secondaires et les espaces anciennement cultivés.

Les richesses spécifiques moyennes des espèces arborescentes des différents habitats ne présentent pas de différence significative. Ce résultat est contraire à ceux de Vroh et Adou Yao (2018) dont les résultats de recherche ont montré que la diversité des espèces était significativement plus élevée dans les forêts anciennes. Cela s'explique par le fait que dans le cas des jachères, nous avons considéré toutes les espèces arborescentes rencontrées dans ces biotopes hormis les cultures principales. Les jachères et les forêts secondaires se reconstituent en grande partie grâce à la banque de graine contenue dans le sol. Cette banque de graine provient de celle de la végétation environnante c'est-à-dire des forêts anciennes. Aussi Adingra et Kassi (2016) ont montré que cette succession est accompagnée de l'augmentation de la richesse spécifique des jachères en fonction de leur âge d'abandon. Aussi dans le cas des jachères, compte tenu du statut de protection du parc, les paysans dissimulaient leurs plantations de cultures pérennes dans le couvert forestier existant. Ainsi, des espèces de forêts anciennes sont présentes dans ces biotopes (Koffi et al., 2015). Ces données obtenues traduisent le niveau de reconstitution élevé des forêts secondaires et des jachères issues de cultures cacaoyères, caféières, de palmier à huile, d'hévéa.

Dans les forêts anciennes, la majorité des espèces ont des feuilles macrophylles et mésophiles, se disséminent par la zoochorie et sont des espèces d'ombre. Cette dominance des espèces d'ombre à feuilles macrophylles et mésophiles et à dissémination zoochore est aussi rencontrée dans les forêts secondaires et les jachères. Cette importance des espèces à feuille macrophylles et mésophylles s'explique par le fait que nos inventaires ont porté sur des espèces phanérophytes à dominance d'espèces arbustives qui sont généralement macrophylles et mésophylles (Bangirinama et al., 2010). En outre, les espèces qui composent les familles des Rubiaceae, Moraceae, Malvaceae, Euphorbiaceae, Fabaceae, Annonaceae qui sont les plus importantes dans les différents biotopes étudiés, ont principalement des feuilles mésophylles, des microphylles et des macrophylles. Ce résultat est similaire à celui de Lumengo et al. (2018) dans la réserve forestière de l'Inera Kiyaka (province de Kwilu) au Congo. De même, la faible proportion d'espèces microphylles observée dans ces milieux signifie que ceux-ci tendent à avoir une canopée fermée. En effet, pour faire face aux conditions d'éclairement élevé, les espèces réduisent au maximum leur surface limbaire exposée en vue de limiter leur perte en eau (Kikufi et Lukoki, 2008).

L'importance de la zoochorie dans les formations pourrait s'expliquée par le fait que ces milieux constituent des habitats pour la faune qui entretient des relations avec la flore. Bangirinama et al. (2013) ont souligné que le mode de dissémination le plus fréquent lorsque les forêts secondaires deviennent matures est la zoochorie. Ces formations anthropisées datent des années 1986 et 2000 et constituent aujourd'hui des forêts secondaires matures. Aussi, c'est ce mode de dissémination qui domine dans les forêts tropicales humides comme celle de la Guyane (Reira et al., 1989), du Cameroun (Ngueguim et al., 2016), du bassin du Congo (Sonke, 1998 ; Kimpouni, 2017) et de certaines forêts en Côte d'ivoire (N'Guessan et Kassi, 2018 ; Kassi et al., 2012). L'abondance des espèces anémochores après est due au fait que ces milieux ont été très ouverts autrefois et par conséquent soumis aux vents. Bangirinama et al. (2010) ont souligné que ces espèces anémochores sont plus importantes durant les premiers stades de reconstitution. Dans les forêts anciennes, ce fait est imputable aux perturbations naturelles telles que les chablis qui conduisent à des ouvertures dans l'habitat. La faible proportion d'espèces barochores a été aussi relevée par Kassi et al. (2017) dans la forêt classée de la Besso. L'importance des 
espèces sciaphiles dans les différents milieux est le reflet de la fermeture de la canopée de ceux-ci. Les espèces pionnières héliophiles, présentes aux débuts de l'ouverture de ces milieux, diminuent progressivement au profit des espèces d'ombre qui sont tolérant à l'absence de lumière lors de cette fermeture de la canopée.

Les modes de dissémination, les types foliaires et la tolérance à la lumière de la plupart des espèces des jachères et des forêts secondaires égalent ceux observés dans les forêts anciennes. Ce résultat témoigne par conséquent d'un rétablissement de l'organisation fonctionnel de ces écosystèmes perturbés. Selon Guariguata et Ostertag (2001), un écosystème perturbé peut recouvrir son fonctionnement longtemps avant que la composition floristique initiale ne soit entièrement restaurée.

Les activités anthropiques, agissant comme des filtres environnementaux, ont entrainé une variation d'abondance relative moyenne des espèces pionnières, des espèces à feuilles mégaphylles et celles se disséminant par la zoochorie et la barochoorie. Dans les veilles jachères, on assiste à une augmentation de l'abondance relative des espèces mégaphylles et pionnières contrairement aux forêts secondaires issues d'activité de défrichement. Ce résultat confirme celui de Lohbeck et al. (2012) qui ont montré que la diversité fonctionnelle des forêts tropicales change au cours des successions secondaires. Aussi, ces jachères ont pour précédent cultural des cultures cacaoyères, d'hévéas, de caféièrs et de Palmiers à huile. La caféiculture était réalisée en plein soleil. Aussi, lors de la réalisation de la palmeraie, des interlignes sont réalisés entre les individus de palmier à huile. Ce dispositif offrait des troués de lumières qui sont importantes pour le développement des espèces pionnières. A cela s'ajoute le fait que les espèces pionnières telles qu'Albizia adianthifolia (Schumach.) W.F. Wright Xylopia aethiopica (Dunal) A. Rich étaient très abondantes dans ces formations. Dans ces jachères, les espèces mégaphylles notamment Musanga cecropioides $\mathrm{R}$. Br. et Myrianthus arboreus $\mathrm{P}$. Beauv. ont été très abondantes contrairement aux forêts secondaires. L'abondance relative moyenne d'espèces barochores très élevée dans les formations secondaires est due à la forte présence dans ce milieu de l'espèce barochore Tabernaemontana crassa Benth. contrairement aux autres milieux.

\section{Conclusion}

Cette étude avait pour objectif d'évaluer les changements des traits fonctionnels des espèces végétales entre reliques de forêts anciennes, forêts secondaires et jachères d'âges différents du Parc National d'Azagny. Il ressort que la majorité des espèces des forêts anciennes, des forêts secondaires et des jachères sont des espèces d'ombre, avec des feuilles mésophylles, macrophylles et une dissémination zoochore. Ce qui traduit un retour au fonctionnement initial des milieux anthropisés. Cependant, les pratiques agricoles et de gestion réalisées auparavant dans le Parc National d'Azagny ont un impact sur la composition fonctionnelle de ces milieux par l'analyse de la distribution des abondances relative des traits. En effet, des différences significatives au niveau des modalités de trait mégaphylles, pionnière, zoochorie et barochorie ont été révélées. Dans les jachères, les individus mégaphylles et pionnières ont été plus abondantes. Dans les forêts secondaires issues de défriche, ce sont les individus barochores. Aussi, les abondances relatives moyennes des espèces zoochores entre les forêts secondaires et les jachères et entre les forêts secondaires et les forêts anciennes sont significativement différentes. Un renforcement des mesures de protection permettra de faciliter la reconstitution de ce milieu naturel après ces perturbations anthropiques.

\section{CONFLIT D'INTERETS}

Les auteurs déclarent qu'ils n'ont aucun conflit d'intérêts.

\section{CONTRIBUTIONS DES AUTEURS}

Dans la présente étude, l'auteur AEGA a élaboré le protocole de recherche comprenant la collecte et les analyses de données de même que la rédaction du 
manuscrit. Les auteurs HSDK et SFD, nous ont apporté une aide précieuse dans la collecte des données. L'auteur BTAV a apporté des corrections et des suggestions au protocole de recherche comprenant la collecte et les analyses de données de même qu'à la rédaction du manuscrit. BTAV a aussi participé à la lecture et à la correction du manuscrit. Les auteurs DK, BZBG, AG et BJCK ont supervisé la collecte des données sur le terrain. L'auteur CYAY est celui qui nous a permis d'obtenir le financement grâce au projet SEP2D-PRP3-45. En outre, il a supervisé la conception du protocole de recherche, la collecte, le traitement des données et la rédaction du manuscrit final.

\section{REMERCIEMENTS}

Nous adressons nos remerciements à l'Office Ivoirien des Parcs et Réserves (OIPR) pour avoir autorisé l'accès dans le Parc National d'Azagny.

\section{REFERENCES}

Adingra OMMA, Kassi JN. 2016. Diversité floristique, structure et dynamique des jachères post-culturales de la forêt classée de Bamo (Côte d'Ivoire). Agronomie Africaine, 28(1): 24-32. DOI : https://www.ajol.info/index.php/aga/issu e/view/16423

Bangirinama F, Bigendako MJ, Lejoly J, Noret $\mathrm{N}$, De Cannière $\mathrm{C}$, Bogaert $\mathrm{J}$. 2010. Les indicateurs de la dynamique post-culturale de la végétation des jachères dans la partie savane de la réserve naturelle forestière de Kigwena (Burundi). Plant Ecology and Evolution, 143(2): $\quad 138-147 . \quad$ DOI: https://doi.org/10.5091/plecevo.2010.386

Bangirinama F, Masharabu T, Havyarimana F. 2013. Analyse des traits biologiques au cours de la dynamique post-culturale : cas des jachères de la zone périphérique de la Réserve naturelle forestière de Bururi. Bulletin Scientifique de l'Institut National pour l'Environnement et la Conservation de la Nature, 12: 61-69. DOI : https://bi.chmcbd.net/biodiversity/bulletin- scientifique/propos-du-bulletinscientifique-de-l-inecn.

Brakchi-Ouakour L, Kadik L, Gachet S. 2015. Typologie fonctionnelle et taxinomique des pinèdes de l'algérois et de l'oranais (Algérie). Revue d'Ecologie (Terre et Vie), 70(3): 231-247. DOI: https://doi.org/10.19182/bft2018.337.a31 633

Brice MH. 2015. Impacts de l'urbanisation sur la diversité spécifique et fonctionnelle dans les forêts riveraines. Mémoire de Maîtrise, Université de Montréal, Canada, $97 \mathrm{p}$.

Densereaux P, Lems K. 1957. The grading of dispersal types in plant communities and their ecological significance. Institut Botanique de l'Université de Montréal, $52 \mathrm{p}$.

Diaz S, Cabido M. 2001. Vive la différence: plant functional diversity matters to ecosystem processes. Tendances en Ecologie et Evolution, 16(11): 646-655. DOI: $\quad$ https://doi.org/10.1016/s01695347(01)02283-2

Flores O. 2005. Déterminisme de la régénération chez quinze espèces d'arbres tropicaux en forêt guyanaise : les effets de l'environnement et de la limitation par la dispersion. Thèse de doctorat, Université Montpellier II, France, 208 p.

Garnier E, Lavorel S, Ansquer P, Castro H, Cruz P, Dolezal J, Eriksson O, Fortunel C, Freitas H, Golodets C, Grigulis K, Jouany C, Kazakou E, Kigel J, Kleyer M, Lehsten V, Leps J, Meier T, Pakeman R, Papadimitriou M, Papanastasis VP, Quested H, Quetier F, Robson M, Roumet C, Rusch G, Skarpe C, Sternberg M, Theau JP, Thebault A, Vile D, Zarovali MP. 2007. Assessing the effects of land-use change on plant traits, communities and ecosystem functioning in grasslands: a standardized methodology and lessons from an application to 11 European sites. Annals of Botany, 99: 967-985. DOI: https://doi.org/10.1093/aob/mcl215

Giller PS, Hillebrand H, Berninger U-G, Gessner MO, Hawkins S, Inchausti P, 
Inglis C, Malmqvist HLB, Michael T, Monaghan TM. 2004. Biodiversity effects on ecosystem functioning: emerging issues and their experimental test in aquatic environments. Oikos, 104: 423-436.

DOI:

https://doi.org/10.1111/j.0030-

1299.2004.13253.x

Gnagbo A, Kouamé D, Adou Yao CY. 2016. Diversité des épiphytes vasculaires de la strate inférieure des formations végétales du Parc National d'Azagny (Sud de la Côte d'Ivoire). Journal of Animal \& Plant Sciences, 28(1): 4366-4386. https://m.elewa.org/Journals/about-japs/.

Guariguata MR, Ostertag R. 2001. Neotropical secondary forest succession: changes in structural and functional characteristics. Forest Ecology and Management, 148(1-3): 185-206. DOI: https://doi.org/10.1016/S0378-1127 (00)00535-1

Hall JB, Swaine MD. 1981. GeobotanyDistribution and Ecology of Vascular Plants in a Tropical Rainforest Forest Vegetation in Ghana. The Hague. DOI: https://doi.org/10.2307/4110055

Hawthorne W, Jongkind C. 2006. Woody plants of western African forests. A guide to the forest trees, shrubs and lianes from Senegal to Ghana, $521 \mathrm{p}$.

Kassi NJ, Yongo OD, Ake Assi A, Decocq G. 2011. L'intérêt d'une approche systémique des dynamiques de végétations en phytosociologie forestière tropicale: cas de la forêt classée de Sanaimbo à Bongouanou/Dimbokro (Côte d'Ivoire). International Journal of Biological and Chemical Sciences, 5(3): 1337-1350. DOI: http://dx.doi.org/10.4314/ijbcs.v5i3.7228 8

Kassi JN, Kouassi RH, Yongo DO. 2012. Analyse de la flore de la forêt classée de Sanaimbo à Bongouanou-Dimbokro (Côte d'Ivoire). International Journal of Biological and Chemical Sciences, 6(5): 2139-2148.

DOI: http://dx.doi.org/10.4314/ijbcs.v6i5.21

Kassi JN, Tuo Y, Zo-Bi IC. 2017. Diversité floristique et infiltration humaine de la forêt classée de la Besso (Côte d'Ivoire). Journal of Applied Biosciences, 114(1): 11299-11308.

DOI: http://dx.doi.org/10.4314/jab.v114i1.3

Kikufi BA, Lukoki FL. 2008. Etude floristique et écologique des marais de Masina. Revue Congolaise des Sciences Nucléaires, 23: $1-20$. https://www.academia.edu/8827362/.

Kimpouni V. 2017. Phytoécologie des faciès à Marantaceae de la forêt de Lossi (Congo, Brazzaville). International Journal of Biological and Chemical Sciences, 11(4): $\quad$ 1437-1460. $\quad$ DOI: http://dx.doi.org/10.4314/ijbcs.v11i4.4

Klais R, Norros V, Lehtinen S, Tamminen T, Olli K. 2016. Community assembly and drivers of phytoplankton functional structure. Functional Ecology, 31(3): 760-767.

DOI: https://doi.org/10.1111/1365-2435.12784

Koffi KAD, Adou Yao CY, Vroh BTA, Gnagbo A, N'Guessan KE. 2015. Diversités floristique et structurale des espaces anciennement cultivés du Parc National d'Azagny (Sud de la Côte d'Ivoire). European Journal of Scientific Research, 134(4): 415-427. https://www.researchgate.net/publication 1283119483.

Konan HDS. 2017. Evolution des types d'occupation du sol dans le parc national d'Azagny (sud de la côte d'ivoire) de 1985 à 2018. Mémoire de Master de Biodiversité et Gestion Durable des Ecosystèmes (Option Ecologie et Conservation Durable des Ecosystèmes), UFR Environnement, Université Jean Lorougnon Guede, Daloa, Côte d'Ivoire, $49 \mathrm{p}$.

Lohbeck L, Poorter L, Paz H, Pla L, Van Breugel M, Martínez-Ramos M, Bongers F. 2012. La diversité fonctionnelle change au cours de la succession des forêts tropicales. Perspectives en Ecologie Végétale, Evolution et Systématique, 14(2): $\quad$ 89-96. DOI: 10.1016/j.ppees.2011.10.002.

Lohbeck MWM. 2014. Écologie fonctionnelle du rétablissement de la forêt 
tropicale. Thèse de doctorat, Université de Wageningen, Wageningen, $224 \mathrm{p}$.

Lumengo AM, Belesi KH, Lubini AC, Kidikwadi TE. 2018. Etude préliminaire sur la flore de la réserve forestière de l'Inera Kiyaka (province de Kwilu). International Journal of Innovation and Applied Studies, 23(4): 474-487. http://www.ijias.issrjournals.org/fr/.

Mekideche S, Brakchi-Ouakour L, Kadik L. 2018. Impact des perturbations anthropiques sur la diversité végétale de la subéraie de Chréa, au nord de 1'Algérie. Bois \& Forets des Tropiques, 337: 53-66. DOI: https:// doi.org/10.19182/bft2018.337.a31633

Mirabel A. 2018. Réponse et Résilience de la Biodiversité d'une Forêt Tropicale après Perturbation. Thèse de doctorat en Sciences et Technologies, Université de Guyane, France, 100 p.

Mouton JA. 1966. Les types biologiques foliaires de Raunkiaer : Etat actuel de la question. Bulletin de la Société Botanique de France, 113(2): 28-36. DOI : https://doi.org/10.1080/00378941.1966.1 0838471

Ngueguim JR, Zapfack L, Onana JM, Noiha NV, Onana D, Betti JL, Riera B. 2016. Can the Mangombe forest plantation contribute to restore and conserve biodiversity? International Journal of Biological and Chemical Sciences, 10(1): $\quad 355-368 . \quad$ DOI: http://dx.doi.org/10.4314/ijbcs.v10i1.27

N'guessan AE, Kassi NJ. 2018. Analyse de la diversité floristique de la forêt classée D'Agbo I (Côte d'Ivoire). European Scientific Journal, 14(9): 346-357. DOI: http://dx.doi.org/10.19044/esj.2018.v14n 9 p346

Payeur ME. 2010. Effets des pratiques sylvicoles sur les traits fonctionnels des communautés végétales de friches arbustives. Mémoire présenté à la Faculté des études supérieures en vue de l'obtention du grade de Maître ès sciences (M.Sc.) en sciences biologiques, Université de Montréal, Canada, 40 p.

Poorter L, Bongers F, Kouamé FN, Hawthorne WD. 2003. Biodiversity of West African Forests and Ecological. Atlas of Woody Plant Species.

Puig H. 2001. Diversité spécifique et déforestation : l'exemple des forêts tropicales humides du Mexique. Bois et Forêts des Tropiques, 268(2): 51-55. DOI:

https://doi.org/10.19182/bft2001.268.a20 102

Raunkiaer C. 1934. The lifes forms of plants and statistical plant geography. In The Life Forms of Plants And Statistical Plant Geography. Oxford University Press: London.

Riéra B, Puig H, Lescure JP. 1989. La dynamique de la forêt naturelle. Bois \& Forets des Tropiques, 219(219): 69-78. DOI : https://doi.org/10.19182/bft1989.219.a19 648

Sonke B. 1998. Études floristiques et structurales des forêts de la réserve de faune du Dja (Cameroun). Thèse de Doctorat, Université Libre Bruxelles, Belgique, $267 \mathrm{p}$.

UICN/BRAO. 2008. Evaluation de l'efficacité de la gestion des aires protégées : Parc et Réserve de Côte d'ivoire. UICN/BRAO, $42 \mathrm{p}$.

Violle C, Navas ML, Vile D, Kazakou E, Fortunel C, Hummel I, Garnier E. 2007. Let the concept of trait be functional. Oikos, 116(5): 882-892. DOI: https://doi.org/10.1111/j.0030-

1299.2007.15559.x

Vroh BTA, Adou Yao CYA. 2018. Successional dynamics of tree species during forest recovery in the southeast of Côte d'Ivoire. Sciences de la Vie, de la Terre et Agronomie, 5(2): 30-38. https: //publication.lecames.org/index.php/svt. 